\title{
X-ray Anomalous Scattering of Diluted Magnetic Oxide Semiconductors: Possible Evidence of Lattice Deformation for High Temperature Ferromagnetism
}

\author{
Takeshi Matsumura,, , - Daisuke Okuyama, ${ }^{1}$ Shinya Niioka, ${ }^{1}$ Hideaki Ishida, ${ }^{1}$ Tadashi Satoh, ${ }^{1}$ Youichi \\ Murakami, ${ }^{1}$ Hidemi Toyosaki, ${ }^{2}$ Yasuhiro Yamada, ${ }^{2}$ Tomoteru Fukumura,${ }^{2}$ and Masashi Kawasaki ${ }^{2}, 3$ \\ ${ }^{1}$ Department of Physics, Graduate School of Science, Tohoku University, Sendai, 980-8578, Japan \\ ${ }^{2}$ Institute for Materials Research, Tohoku University, Sendai, 980-8577, Japan \\ ${ }^{3}$ CREST, Japan Science and Technology Agency, Tokyo 102-0075, Japan
}

(Dated: October 28, 2018)

\begin{abstract}
We have examined whether the Co ions crystallographically substitute on the Ti sites in rutile and anatase $\mathrm{Ti}_{1-x} \mathrm{Co}_{x} \mathrm{O}_{2-\delta}$ thin films that exhibit room-temperature ferromagnetism. Intensities of the x-ray Bragg reflection from the films were measured around the $K$-absorption-edge of Co. If the Co ions randomly substitute on the $\mathrm{Ti}$ sites, the intensity should exhibit an anomaly due to the anomalous dispersion of the atomic scattering factor of Co. However, none of the anatase and rutile samples did exhibit an anomaly, unambiguously showing that the Co ions in $\mathrm{Ti}_{1-x} \mathrm{Co}_{x} \mathrm{O}_{2-\delta}$ are not exactly located at the $\mathrm{Ti}$ sites of $\mathrm{TiO}_{2}$. The absence of the anomaly is probably caused by a significant deformation of the local structure around Co due to the oxygen vacancy. We have applied the same method to paramagnetic $\mathrm{Zn}_{1-x} \mathrm{Co}_{x} \mathrm{O}$ thin films and obtained direct evidence that the Co ions are indeed substituted on the $\mathrm{Zn}$ sites.

PACS numbers: 75.50.Pp, 78.70.Ck, 68.35.Dv
\end{abstract}

\section{INTRODUCTION}

Diluted magnetic semiconductors (DMS) have been attracting considerable attention as a promising candidate material for spintronics devices. The basic concept is to utilize spin polarized carriers created by strong exchange interaction between localized $d$-electrons of diluted magnetic ions and itinerant $s p$-band electrons $\frac{1,2}{2}$ Since the discovery of room temperature ferromagnetism in Codoped anatase and rutile $\mathrm{TiO}_{2}$ thin film,,$\underline{3}$ there have been a number of reports on high- $T_{C}$ ferromagnetism observed in various kinds of DMS, particularly in oxides $\underline{5, \underline{6}}$ Co-doped $\mathrm{TiO}_{2}$ is the material that has been studied most intensively. However, the origin of the ferromagnetism has not yet been elucidated. One of the experimental issues is whether the ferromagnetism indeed originates from the Co spins that are randomly substituted on the Ti sites, and not from the segregated Co clusters. There have also been a number of reports that ascribe the ferromagnetism to the Co segregation $\underline{7.8,9}$

Strong evidence in support of intrinsic ferromagnetism has been given by the observations of anomalous Hall effect (AHE) and magnetic circular dichroism (MCD) $10,11,12,13,14$ If the ferromagnetism were intrinsic, the charge carriers should be spin polarized due to the exchange interaction with the Co spins. AHE and MCD are considered to probe the ferromagnetic response of the carriers introduced by the oxygen deficiency. In addition, rutile $\mathrm{Ti}_{1-x} \mathrm{Co}_{x} \mathrm{O}_{2-\delta}$ has already been functionalized as a spin tunneling junction working up to 180 $\mathrm{K} .15$ Other studies that focus on this issue are mainly based on spectroscopic measurements such as x-ray absorption fine structure (XAFS) for samples in which no Co segregation was recognized by imaging probes such as scanning electron microscope (SEM), atomic force microscope (AFM), and transmission electron microscopy
(TEM) 16,17,18,19 In these spectroscopic measurements, the spectral line shapes differ from that of Co metal. They state that the local structure of the Co site is close to those of $\mathrm{Co}$ oxides such as $\mathrm{CoTiO}_{3}$, in which the $\mathrm{Co}$ ion is surrounded by six oxygen atoms, forming a $\mathrm{CoO}_{6}$ cluster, and that the valence state of $\mathrm{Co}$ is divalent. Cui et al. performed electron beam diffraction and composition analysis for anatase $\mathrm{TiO}_{2}$ in a selected area without Co segregation and confirmed both the diffraction peaks of $\mathrm{TiO}_{2}$ and the existence of $\mathrm{Co} \stackrel{20}{\underline{20}}$ These results seem to support that the Co ions randomly substitute on the $\mathrm{Ti}$ sites.

Although these spectroscopic methods provide valuable information on the local structure around Co, however, they give little information on the crystallographic position of $\mathrm{Co}$ and the orientation of the $\mathrm{CoO}_{6}$ cluster: the former could be interstitial and the latter could be deformed due to the oxygen vacancy. In this context, we still do not have unambiguous evidence that Co indeed substitutes for $\mathrm{Ti}$ in a crystallographic sense. The fact that Co is not soluble in $\mathrm{TiO}_{2}$ in a thermodynamically stable manner also casts doubt on the assumption that Co substitutes for Ti ${ }^{21}$ From a theoretical point of view, knowledge on the local structure of a Co ion is of fundamental importance to construct a valid model and proceed the calculations on the electronic states of $3 d$ and $s p$-band electrons.

In the present paper, we report on the results of x-ray diffraction utilizing anomalous scattering from Co. This is a fundamentally different approach than spectroscopy. We observe a Bragg peak as a result of the interference among the $\mathrm{x}$ rays scattered from many Co ions in the sample and discuss the average crystallographic site of Co in the $\mathrm{TiO}_{2}$ lattice. If Co substitute exactly on the Ti site, the Co ions have the same periodicity as the $\mathrm{TiO}_{2}$ lattice and contribute to the Bragg peak. Our results on 
anatase and rutile Co-doped $\mathrm{TiO}_{2}$ films, however, lead us to a conclusion that the Co ions are not exactly located on the Ti sites, implying a significant lattice deformation. On the other hand, it is shown that Co indeed substitutes on the $\mathrm{Zn}$ sites in paramagnetic Co-doped $\mathrm{ZnO}$ thin films.

\section{EXPERIMENT}

The basic principle of the method is simple and direct. If the Co ions of concentration $x$ randomly substitute on the $\mathrm{Ti}$ sites of $\mathrm{TiO}_{2}$, the unit-cell structure-factor can be expressed as

$$
\begin{aligned}
F= & \sum_{i}\left\{(1-x) f_{\mathrm{Ti}}+x f_{\mathrm{Co}}\right\} \exp i \boldsymbol{\kappa} \cdot \boldsymbol{R}_{i}^{(\mathrm{Ti})} \\
& +\sum_{i} f_{\mathrm{O}} \exp i \boldsymbol{\kappa} \cdot \boldsymbol{R}_{i}^{(\mathrm{O})},
\end{aligned}
$$

where $f_{\mathrm{Ti}}, f_{\mathrm{Co}}$, and $f_{\mathrm{O}}$ are the atomic scattering factors of Ti, Co, and O, respectively. $\boldsymbol{R}_{i}^{(\mathrm{Ti})}$ and $\boldsymbol{R}_{i}^{(\mathrm{O})}$ represent the $i$-th atomic site of $\mathrm{Ti}$ and $\mathrm{O}$ in the unit cell, respectively. $\kappa$ is the scattering vector. Here, the atomic scattering factors are energy dependent and are generally expressed as

$$
f(E)=f^{0}+f^{\prime}(E)+i f^{\prime \prime}(E),
$$

where $f^{0}$ is the Thomson scattering factor and $f^{\prime}$ and $f^{\prime \prime}$ are the real and imaginary parts, respectively, of the anomalous scattering factor, which exhibit a significant anomaly near an absorption edge of the element. Therefore, when we measure the energy dependence of the intensity of a Bragg reflection from the Co-doped $\mathrm{TiO}_{2}$ film, the intensity should exhibit an anomaly at the absorption edge of Co if the structure factor involves $f_{\mathrm{Co}}$. This measurement can be performed using a synchrotron radiation source, where the incident energy of the $\mathrm{x}$ rays can be varied. Information of the crystal structures, reflection indices examined in the present experiment, and their structure factors are listed in Table [ In rutile, the $\mathrm{Ti}$ atoms occupy the crystallographic site of $2 a:(0,0,0)$ and $\left(\frac{1}{2}, \frac{1}{2}, \frac{1}{2}\right)$. In anatase, they are at the $4 a$ site: $(0,0$, $0),\left(0, \frac{1}{2}, \frac{1}{4}\right),\left(\frac{1}{2}, \frac{1}{2}, \frac{1}{2}\right)$, and $\left(\frac{1}{2}, 0, \frac{3}{4}\right)$. The $\mathrm{Zn}$ atoms of $\mathrm{ZnO}$ occupy the $2 b$ site of the wurtzite structure: $\left(\frac{1}{3}, \frac{2}{3}\right.$, $0)$ and $\left(\frac{2}{3}, \frac{1}{3}, \frac{1}{2}\right)$.

We used the same rutile $\mathrm{Ti}_{1-x} \mathrm{Co}_{x} \mathrm{O}_{2-\delta}$ epitaxial thin film samples as those studied in Refs. 10 and 11. The films were deposited on $\mathrm{TiO}_{2}$ buffered r-sapphire substrates by laser molecular beam epitaxy (MBE). Oxygen deficiency $\delta$ is controlled by the oxygen partial pressure varying from $10^{-4}$ to $10^{-8}$ Torr. A systematic relationship among Co concentration $x$, oxygen partial pressure, carrier density, conductivity, ferromagnetic moment, AHE, and MCD is well established for these samples. Results of x-ray photoemission spectroscopy (XPS) and XMCD at the Co $L_{2,3}$-edges for these samples are also reported, $\stackrel{22,23}{\rightleftharpoons}$ both of which conclude that the spectrum is that of a high-spin $\mathrm{Co}^{2+}$ ion in a crystal field of oxygen octahedron and that the room-temperature ferromagnetism is intrinsic. Anatase $\mathrm{Ti}_{1-x} \mathrm{Co}_{x} \mathrm{O}_{2-\delta}$ epitaxial thin film was deposited on $\mathrm{LaAlO}_{3}-(001)$ substrate by laser MBE in an oxygen partial pressure of $1 \times 10^{-6}$ Torr and at a growth temperature of $700{ }^{\circ} \mathrm{C}$. The appearance of room-temperature ferromagnetism was checked by a SQUID magnetometer and no Co segregation was recognized by AFM and SEM. $\mathrm{Zn}_{1-x} \mathrm{Co}_{x} \mathrm{O}$ epitaxial thin films are the same as those of Ref. 24. These exhibit large MCD without an appearance of ferromagnetism, although several studies reported high- $T_{C}$ ferromagnetism in the same compound,,$\underline{5} \cdot \underline{6}$

$\mathrm{X}$-ray diffraction experiments were performed using four-circle diffractometers installed at beamlines 1A, 4C, and $16 \mathrm{~A} 2$ of the Photon Factory in KEK, Japan. The incident beam was monochromatized by a $\mathrm{Si}-111$ double crystals and focused by a bent cylindrical mirror. The energy was calibrated using the absorption edge of a Co metal foil. For each thin-film sample, we first measured the fluorescence spectrum near the Co $K$-edge to check if Co was actually included in the area where the beam was irradiated. Next, we measured the energy dependence of the intensity of the Bragg reflection. The typical beam size was $\sim 1 \times 1 \mathrm{~mm}^{2}$. All the measurements were carried out at room temperature.

\section{EXPERIMENTAL RESULTS}

The results for $\mathrm{Zn}_{1-x} \mathrm{Co}_{x} \mathrm{O}$ with $x=0.02$, 0.04, and 0.12 are shown in Fig. 1. The base line of each spectrum is normalized to unity. The step in the fluorescence at 7.72 $\mathrm{keV}$, the $K$-edge of $\mathrm{Co}$, is roughly proportional to the Co concentration, indicating that Co is actually included in the irradiated area with concentrations proportional to the nominal value. Energy dependence of the intensity of the 002 Bragg reflection also exhibits a clear anomaly at the $K$-edge. This directly indicates that the Co ions indeed substitute on the $\mathrm{Zn}$ sites randomly. Comparison with the calculated curve represented by the lines is also satisfactory $\underline{25}$

$\mathrm{Ti}_{1-x} \mathrm{Co}_{x} \mathrm{O}_{2-\delta}$ samples exhibit contrasting results with $\mathrm{Zn}_{1-x} \mathrm{Co}_{x} \mathrm{O}$ as described in the following. Figure 2 shows the results for anatase $\mathrm{Ti}_{1-x} \mathrm{Co}_{x} \mathrm{O}_{2-\delta}$ with a nominal concentration of $x=0.05$. The base lines are normalized to unity. The fluorescence spectrum indicates that the Co ions are indeed included in the sample. However, the intensity of the 004 Bragg reflection does not exhibit any anomaly at the absorption edge of Co. If the Co ions with $x=0.05$ substitute for $\mathrm{Ti}$, an anomaly as demonstrated by the solid line is expected, which is as large as about $5 \%$ of the anomaly actually observed at the $K$ edge of $\mathrm{Ti}$ as shown in the inset. These results mean that the doped Co ions are not located exactly on the Ti sites, although the Co ions indeed exist in the sample.

Figure 3 shows the results for rutile $\mathrm{Ti}_{1-x} \mathrm{Co}_{x} \mathrm{O}_{2-\delta}$ with nominal concentrations of $x=0.05$ and 0.1 , grown under an oxygen partial pressure of $10^{-7}$ Torr. The flu- 
TABLE I: Reflection index and the structure factor examined in this experiment.

\begin{tabular}{lllll}
\hline \hline sample & structure & space group & index & structure factor \\
\hline $\mathrm{Ti}_{1-x} \mathrm{Co}_{x} \mathrm{O}_{2}$ & anatase & $I 4_{1} /$ amd $(\# 141)$ & 004 & $F=4(1-x) f_{\mathrm{Ti}}+4 x f_{\mathrm{Co}}+8 f_{\mathrm{O}} \cos 1.66 \pi$ \\
$\mathrm{Ti}_{1-x} \mathrm{Co}_{x} \mathrm{O}_{2}$ & rutile & $P 4_{2} / m n m(\# 136)$ & 202 & $F=2(1-x) f_{\mathrm{Ti}}+2 x f_{\mathrm{Co}}+4 f_{\mathrm{O}} \cos 1.22 \pi$ \\
$\mathrm{Zn}_{1-x} \mathrm{Co}_{x} \mathrm{O}$ & wurtzite & $P 6_{3} m c(\# 186)$ & 002 & $F=2(1-x) f_{\mathrm{Zn}}+2 x f_{\mathrm{Co}}+2 f_{\mathrm{O}}(\cos 1.53 \pi+i \sin 1.53 \pi)$ \\
\hline \hline
\end{tabular}

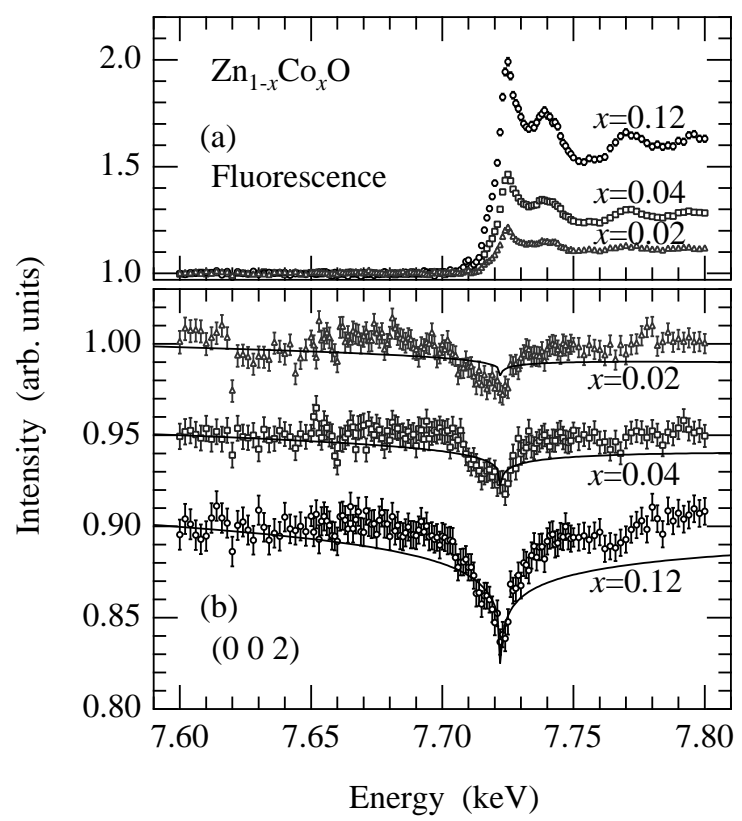

FIG. 1: (a) Fluorescence spectra of $\mathrm{Zn}_{1-x} \mathrm{Co}_{x} \mathrm{O}$ as a function of Co concentration $x$. (b) X-ray energy dependences of the intensity of the 002 Bragg reflection. Data are shifted for $x=0.04$ and 0.12 . Lines represent the calculated curves.

orescence spectra show that the Co ions indeed exist in the samples with actual concentrations proportional to the nominal value. However, as in anatase, the intensities of the 202 Bragg reflection do not exhibit any anomaly at the absorption edge, even in the high concentration sample of $x=0.1$. These results again mean that the doped Co ions are not exactly on the $\mathrm{Ti}$ sites, although the Co ions indeed exist in the film. Since we expect an anomaly as large as the one demonstrated by the solid line in Fig. 3, the actual amount of substitution, if any, is estimated to be much less than $1 \%$ both for $x=0.05$ and 0.1 . The measurements on other reflections, e.g., 103 for anatase and 101 for rutile, and also on a few other samples, did not exhibit any anomaly.

\section{DISCUSSIONS}

The present experimental results unambiguously show that the atomic scattering factor of $\mathrm{Co}$ is not included in the structure factor of either anatase or rutile $\mathrm{Ti}_{1-x} \mathrm{Co}_{x} \mathrm{O}_{2-\delta}$. In other words, the Co ions, on aver-

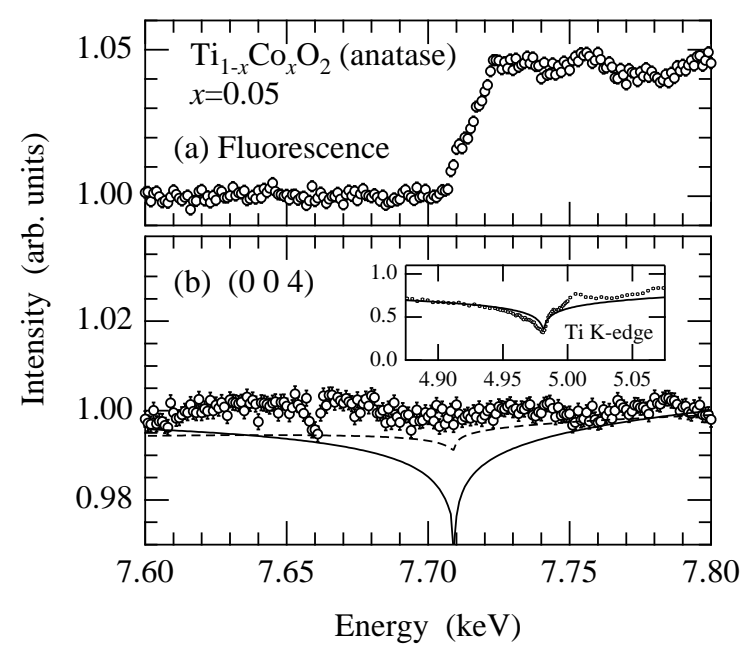

FIG. 2: (a) Fluorescence spectrum of anatase $\mathrm{Ti}_{1-x} \mathrm{Co}_{x} \mathrm{O}_{2-\delta}$ for $x=0.05$. (b) X-ray energy dependence of the intensity of the 004 Bragg reflection. Solid line represents a calculated curve assuming random substitution of $\mathrm{Ti}$ with $5 \%$ Co ions. Dashed line represents a simulation considering local deformations as described in the text. Inset shows the result around the $K$-edge of Ti.

age, do not occupy the Ti site nor any specific crystallographic site in the $\mathrm{TiO}_{2}$ unit cell; therefore, the interference among x-rays scattered from randomly distributed Co ions are prevented. In contrast, from spectroscopic measurements such as XAFS, XPS and XMCD, it is concluded that the local environment of Co is close to that of oxygen octahedron $16,17,18,19,20,22,23$ In addition, the strong correlation among Co concentration $x$, oxygen deficiency $\delta$, conductivity, ferromagnetism, AHE, and MCD investigated in Refs. 10, 11, 12,13,14 support that the carriers are associated with the ferromagnetism originating from the randomly distributed Co ions. Taking all these experimental results into consideration, we speculate that the doped Co ions exist in a locally deformed structure, although they are randomly distributed in the sample without making a Co metal-cluster. When a $\mathrm{Co}^{2+}$ ion is substituted for a $\mathrm{Ti}^{4+}$ in $\mathrm{TiO}_{2}$, an oxygen vacancy is necessarily created to maintain the charge neutrality. As a result, the number of oxygen in the ligands becomes less than six, ${ }^{16}$ which would lead to a deformation of the local structure around Co and a deviation of Co from the exact $\mathrm{Ti}$ site, i.e., the $2 a$ site in rutile and the $4 a$ site in anatase. There is also a possibility that Co substitutes 


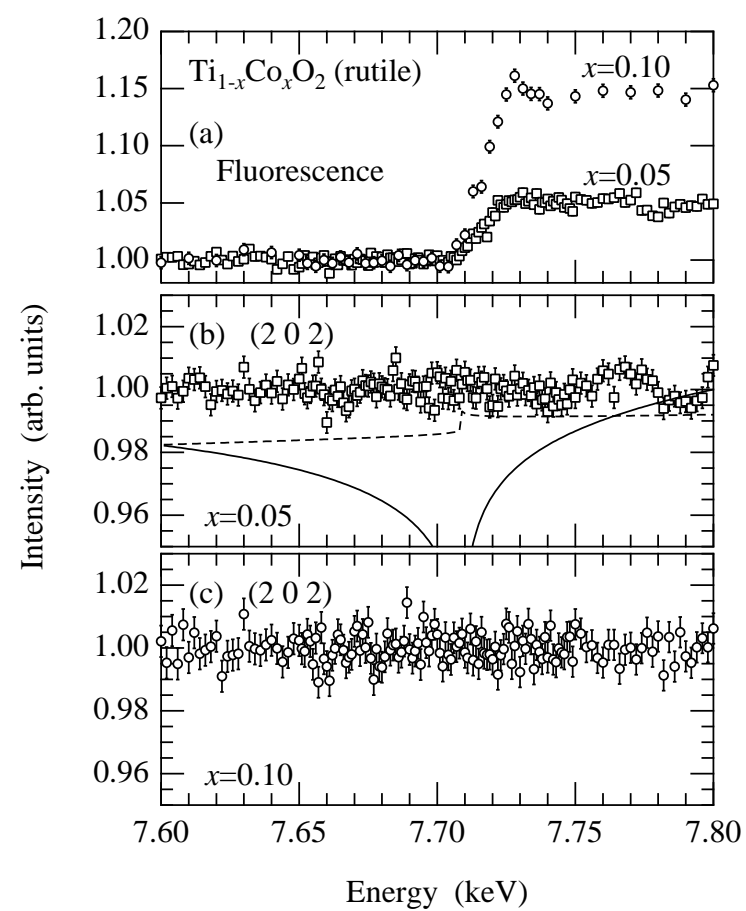

FIG. 3: (a) Fluorescence spectra of rutile $\mathrm{Ti}_{1-x} \mathrm{Co}_{x} \mathrm{O}_{2-\delta}$ for $x=0.05$ and $x=0.1$. (b),(c) X-ray energy dependence of the intensity of the 202 Bragg reflection for $x=0.05$ and $x=$ 0.1. Solid line represents a calculated curve assuming random substitution of Ti with $5 \%$ Co ions. Dashed line represents a simulation considering local deformations as described in the text.

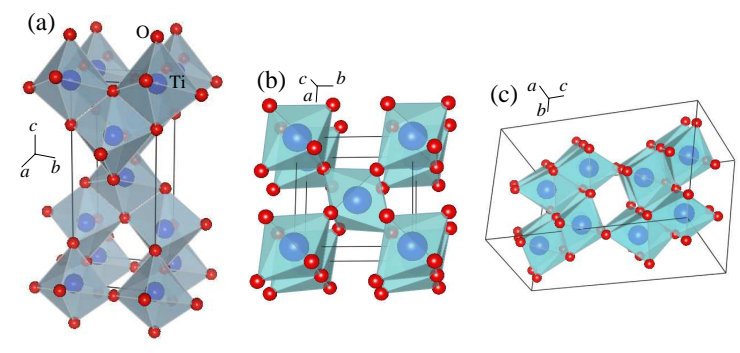

FIG. 4: (Color online) Crystal structure of (a) anatase- $\mathrm{TiO}_{2}$, (b) rutile- $\mathrm{TiO}_{2}$, and (c) $\mathrm{Ti}_{4} \mathrm{O}_{7}$ Magneli phase. ${ }^{26}$

for the interstitial sites among oxygen octahedrons. In rutile $\mathrm{TiO}_{2}$, in particular, the interstitial occupation at positions such as $\left(\frac{1}{2}, 0,0\right),\left(0, \frac{1}{2}, 0\right),\left(\frac{1}{2}, 0, \frac{1}{2}\right)$, and $\left(0, \frac{1}{2}\right.$, $\frac{1}{2}$ ) leads to a structure similar to the Magneli phase as illustrated in Fig. 4, from which we may speculate that the interstitial site could be also stable for Co.

In order to examine if such a deformation could suppress the $K$-edge anomaly of Co, we have made simulations assuming random shifts of the Co ions from the $\mathrm{Ti}$ site or the interstitial site. The result is demonstrated by the dashed lines in Figs. 2 and 3 . The process of the simulation is as follows. First, $5 \%$ of Co is doped into a super cell of $10 \times 10 \times 10$ unit cells, randomly substituting for the $\mathrm{Ti}$ sites in anatase and the interstitial sites in rutile, respectively $\frac{27}{}$ At this stage, the anomaly as demonstrated by the solid lines in Fig. 2 and 3 appears because the Co ions occupy a specific crystallographic site and Eq. (11) is valid. This is the first kind of randomness. Next, we consider the second kind of randomness; i.e., one oxygen vacancy is randomly created in the local octahedron at the Co site, and the Co atom is shifted to the oxygen vacancy by $1 \AA$ in anatase and $0.6 \AA$ in rutile, respectively. Then, we calculate $|F|^{2}$ for the super cell, which is shown by the dashed lines in the figures. Although it is not our intention to emphasize that this is really the case, the second kind of randomness explains that the anomaly at the edge becomes very weak; absence of the anomaly in the 103 reflection of anatase and 101 of rutile can also be explained. In this simulation, the weakened anomaly is associated with the increased number of possible Co sites by a factor six as a result of the second kind of randomness. There should also be a tilting of the octahedron due to the vacancy, which would weaken the anomaly even more because it further increases the possible positions and weakens the correlation among the Co sites; the relatively large shift values assumed above can be reduced. The spectroscopic measurements, which analyze the local structural environment on average, may hide information on this kind of deformation we considered here. In the case of $\mathrm{Zn}_{1-x} \mathrm{Co}_{x} \mathrm{O}$, on the other hand, oxygen vacancy is not necessary when doping because both $\mathrm{Zn}$ and Co are divalent; then, there arises little deformation in the local structure and the doped Co sits exactly on the $\mathrm{Zn}$ site, which is the prerequisite for Eq. (1).

To search for possible deformed structure or another phase that could contain Co such as the Magneli phase, we performed additional x-ray diffraction experiment using an imaging plate Debye-Scherrer camera installed at beamline 1B. However, no other phase was detected as a diffraction peak with its intensity higher than $\sim 0.2 \%$ of the strongest peak of rutile or anatase structure of the $\mathrm{TiO}_{2}$ film. Therefore, the deformed structure, if it existed, is suspected to be short ranged and randomly oriented, which only gives rise to incoherent scattering.

Although the position and the local structure of Co is still uncertain, our experimental results support a theoretical investigation that the oxygen vacancy near Co induces structural deformation and enhances the spin density associated with the ferromagnetism. ${ }^{28}$ A recent theoretical model for high- $T_{C}$ ferromagnetism in oxide DMS is also based on the oxygen vacancy, which causes impurity band exchange. ${ }^{29}$ Elucidation of the microscopic structure around Co and its relation with the mechanism of the ferromagnetism is strongly required. Structural analysis by x-ray fluorescence holography, which determines the three dimensional atomic arrangement around the fluorescing atom, could solve this problem. ${ }^{30}$ 


\section{CONCLUSION}

By utilizing x-ray anomalous dispersion, we have directly examined whether the doped Co ions substitute for $\mathrm{Ti}$ in anatase and rutile $\mathrm{Ti}_{1-x} \mathrm{Co}_{x} \mathrm{O}_{2-\delta}$ for well characterized thin-film samples exhibiting intrinsic high- $T_{C}$ ferromagnetism. Although the intensity of the Bragg reflections should exhibit an anomaly at the $K$-edge of Co if the Co ions were randomly substituted exactly on the Ti site, no anomaly was detected in the experiment, indicating that the Co ions are not located exactly on the Ti site. However, the fluorescence spectra show that the Co ions exist in the sample in a certain form; XPS and XMCD spectra on the identical sample support that the Co ions are randomly distributed and are surrounded by the oxygens. These contrasting results suggest that the local structure around Co is strongly deformed, leading to a significant shift of Co from the high symmetry positions of $\mathrm{Ti}$ sites or interstitials, probably because of the oxygen vacancy. We have proposed a scenario how the anomaly disappears by the local deformations. On the other hand, in our paramagnetic $\mathrm{Zn}_{1-x} \mathrm{Co}_{x} \mathrm{O}$ thin film, the substitution of Co for the $\mathrm{Zn}$ sites has been verified. These results may imply a significant role of lattice deformation for the high- $T_{C}$ ferromagnetism in oxide DMS's.

\section{Acknowledgments}

We wish to acknowledge the technical support of $Y$. Wakabayashi and H. Sawa during the experiments at the photon factory. This work was supported by a Grant-inAid for Scientific Research on Priority Area, "Invention of anomalous quantum materials", from the Ministry of Education, Science, Sports and Culture of Japan. T. F is supported by NEDO, Industrial Research Grant Program (05A24020d).
* tmatsu@iiyo.phys.tohoku.ac.jp

1 T. Dietl, H. Ohno, F. Matsukura, J. Cibert, and D. Ferrand, Science 287, 1019 (2000).

2 H. Ohno, Science 281, 951-956 (1998).

3 Y. Matsumoto, M. Murakami, T. Shono, T. Hasegawa, T. Fukumura, M. Kawasaki, P. Ahmet, T. Chikyow, S.-Y. Koshihara and H. Koinuma, Science 291, 854 (2001).

4 Y. Matsumoto, R. Takahashi, M. Murakami, T. Koida, X.-J. Fan, T. Hasegawa, T. Fukumura, M. Kawasaki, S.Y. Koshihara and H. Koinuma, Jpn. J. of Appl. Phys. 40, L1204 (2001).

5 T. Fukumura, H. Yoyosaki, and Y. Yamada, Semicond. Sci. and Technol. 20, S103 (2005).

6 R. Janisch, P. Gopal, and N. A. Spaldin, J. Phys: Condens. Matter 17, R657 (2005).

7 J.-Y. Kim, J.-H. Park, B.-G. Park, H.-J. Noh, S.-J. Oh, J. S. Yang, D.-H. Kim, S. D. Bu, T.-W. Noh, H.-J. Lin, H.-H. Hsieh, and C. T. Chen, Phys. Rev. Lett. 90, 017401 (2003).

8 S. R. Shinde, S. B. Ogale, J. S. Higgins, H. Zheng, A. J. Millis, V. N. Kulkarni, R. Ramesh, R. L. Greene, and T. Venkatesan, Phys. Rev. Lett. 92, 166601 (2004).

9 J. S. Higgins, S. R. Shinde, S. B. Ogale, T. Venkatesan, and R. L. Greene, Phys. Rev. B 69, 073201 (2004).

10 H. Toyosaki, T. Fukumura, Y. Yamada, K. Nakajima, Y. Chikyow, T. Hasegawa, H. Koinuma, and M. Kawasaki, Nature Mater. 3, 221 (2004).

11 H. Toyosaki, T. Fukumura, Y. Yamada, and M. Kawasaki, Appl. Phys. Lett. 86, 182503 (2005).

12 T. Fukumura, Y. Yamada, K. Tamura, K. Nakajima, T. Aoyama, A. Tsukazaki, M. Sumiya, S. Fuke, Y. Segawa, Y. Chikyow, T. Hasegawa, H. Koinuma, and M. Kawasaki, Jpn. J. Appl. Phys. 42, L105 (2003).

13 Y. Yamada, H. Yoyosaki, A. Tsukazaki, T. Fukumura, K. Tamura, Y. Segawa, K. Nakajima, T. Aoyama, Y. Chikyow, T. Hasegawa, H. Koinuma, and M. Kawasaki, J. Appl. Phys. 96, 5097 (2004).

14 K. Ueno, T. Fukumura, H. Toyosaki, M. Nakano, and M. Kawasaki, Appl. Phys. Lett. 90, 072103 (2007).
15 H. Toyosaki, T. Fukumura, K. Ueno, M. Nakano, and M. Kawasaki, Jpn. J. Appl. Phys. 44, L896 (2005).

16 S. A. Chambers, S. M. Heald, and T. Droubay, Phys. Rev. B 67, 100401(R) (2003).

17 N. Shimizu, S. Sasaki, T. Hanashima, K. Yamawaki, H. Udagawa, N. Kawamura, M. Suzuki, H. Maruyama, M. Murakami, Y. Matsumoto, and H. Koinuma, J. Phys. Soc. Jpn. 73, 800 (2004).

18 K. A. Griffin, A. B. Pakhomov, C. M. Wang, S. M. Heald, and K. M. Krishnan, Phys. Rev. Lett. 94, 157204 (2005).

19 M. Murakami, Y. Matsumoto, T. Hasegawa, P. Ahmet, K. Nakajima, T. Chikyow, H. Ofuchi, I. Nakai, and H. Koinuma, J. Appl. Phys. 95, 5330 (2004).

20 M. L. Cui, J. Zhu, X. Y. Zhong, Y. G. Zhao, and X. F. Duan, Appl. Phys. Lett. 85, 1698 (2004).

21 J. Li, C. H. Sow, X. S. Rao, C. K. Ong, and D. N. Zheng, Euro. Phys. J. B 32, 471 (2003).

22 J. W. Quilty, A. Shibata, J.-Y. Son, K. Takubo, T. Mizokawa, H. Toyosaki, T. Fukumura, and M. Kawasaki, Phys. Rev. Lett. 96, 027202 (2006).

23 K. Mamiya, T. Koide, A. Fujimori, H. Tokano, H. Manaka, A. Tanaka, H. Toyosaki, T. Fukumura, and M. Kawasaki, Appl. Phys. Lett. 89 , 062506 (2006).

24 Z. Jin, T. Fukumura, M. Kawasaki, K. Ando, H. Saito, T. Sekiguchi, Y. Z. Yoo, M. Murakami, Y. Matsumoto, T. Hasegawa, and H. Koinuma, Appl. Phys. Lett. 78, 3824 (2001).

25 Y. Waseda, Novel Application of Anomalous (Resonace) X-ray Scattering for Structural Characterization of Disordered Materials (Springer-Verlag, Heidlberg, 1984). [Online database of $f^{\prime}$ and $f^{\prime \prime}$ ] http://www.tagen.tohoku.ac.jp/general/building/iamp /database/scm/AXS.

26 Figure was drawn with VICS-II software developed by K. Momma and F. Izumi.

27 If Co atoms were substituted for the $\mathrm{Ti}$ site in rutile, disappearance of the anomaly both in 202 and 101 reflections cannot be explained simultaneously in the present model calculation. 
${ }^{28}$ H. Weng, X. Yang, J. Dong, H. Mizuseki, M. Kawasaki, and Y. Kawazoe, Phys. Rev. B 69, 125219 (2004).

29 J. M. D. Coey, M. Venkatesan, and C. B. Fitzgerald, Nature Mater. 4, 173 (2005).
30 S. Hosokawa, T. Ozaki, K. Hayashi, N. Happo, M. Fujiwara, K. Horii, P. Fons, A. V. Kolobov, and J. Tominaga, Appl. Phys. Lett. 90, 131913 (2007). 